\title{
Analysis of the Rigidity of Arc Flexible Hinge with Different Geometrical Parameters
}

\author{
Hongjiang Chen \\ School of Mechanical and Electrical Engineer, Jiangxi Science \& Technology \\ Normal University, Nanchang, Jiangxi 330013, P.R. China \\ hongjiangc@163.com
}

\begin{abstract}
Flexible hinges are widely used in micro robotic. Its rigidity directly influences an organization's terminal localization. Its actual structure geometry size cannot satisfy the theoretical analysis completely in a theoretical supposition condition. In this paper, we analyzed the rotation rigidity of arc flexible hinges in different parameters using finite element software ANSYS. The errors are discovered and compared with theoretical result. An analysis of changes of parameters on the performance of arc flexible hinge was carried out, though the graph of the flexible hinge's parameters and its performance. The key manufacture parameters that affect the performance of arc flexible hinge the most and rules of design are given, which can provide directions of design precision for the flexible hinge.
\end{abstract}

Keywords: arc flexure hinge, finite element analysis, rigidity.

\section{Introduction}

Flexible hinge have some characteristics, such as small volume, without rubs, ceaseless, good rigidity and high sensitivity. With microcomputer electrical system series (MEMS) technical rapidly expanding, flexible hinge are widely applied in the displacement which requests small angular and high-precision rotation, such as gyroscopes, accelerometers, precision instruments and so on. It has broad application prospects in the micron level domain.

The common flexible hinge is in two kinds: beam-shape flexible hinge and arc-shaped flexible hinge. The beam-shaped flexible hinge has a big slewing area, but the movement precision is bad. The arc-shaped flexible hinge's movement precision is high, but the slewing range is relatively small [1]. In order to take into account the movement precision and scope, the following several rotation flexible hinges have been generated: parabolic flexure hinge, an arc flexure hinge and a hyperbola-shaped flexure hinge, etc [2].The properties of flexible hinges are rigidity, precision and stress characteristic, etc. The rigidity performance reflects the stress ability and also manifests movement to a vice-flexible degree. In 1965, Paros et al [3] announced his design development of the circular flexible hinge for the first time, and gave the rigidity formula. Smith et al used the similar method to obtain an elliptic flexible hinge mechanics expression [4]. Nicolae Lodonitu inferred the parabola and the hyperbolic flexible hinge's rigidity formula [5]. 
Wei Xu and Tim King analyzed the rectangular and ellipse flexible hinge's rigidity and rotation precision using the finite element method [6].

In this paper the arc flexible hinge stiffness to different geometrical parameters is analyzed with software ANSYS10.0. Compared with results of theoretical analysis and finite element analysis (FEA), the errors are analyzed. Through the graph of the flexible hinge parameters and its performance, an analysis of changes of parameters on the performance of the arc flexible hinge was carried out. The key manufacture parameters that affect the performances of an arc flexible hinge the most and rules of design are given, which can give directions of design precision for the flexible hinge.

\section{Rigidity Formula of the Arc Flexible Hinge}

An arc flexible hinge, as shown in Figure 1, is a particular type of flexure that consists of a necked down section. Parameters t, h, b are flexible hinge's smallest thickness, height and width, respectively, parameter $\mathrm{R}$ is the radius of arc, and $\theta_{m}$ is the centre angle of arc.

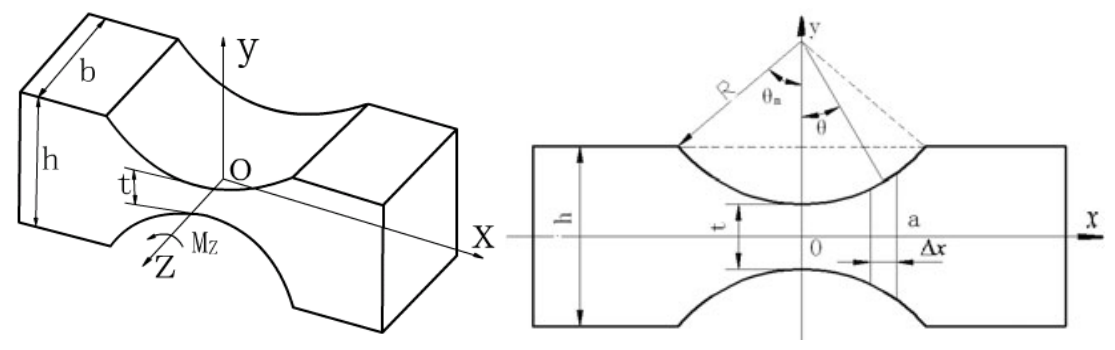

Fig. 1. Model of arc flexible hinge

As shown in Figure 1, the infinitesimal is intercepted in the abscissa axis that is $\Delta x=\Delta(\rho \sin \theta)$. To begin, the infinitesimal section is vertical to the abscissa axis. The flexible hinge's angular deformation $a_{z}$ is generated under torque $\mathrm{M}_{\mathrm{Z}}$ as given in Eq.1 [1].

$$
\begin{gathered}
\alpha_{z}=\int_{-R \sin \theta_{m}}^{R \sin \theta_{m}} \frac{M_{z}(x)}{E I_{z}(x)} d x=\frac{12 M_{z}}{E b R^{2}} \int_{-\theta_{m}}^{\theta_{m}} \frac{\cos \theta}{\left(\frac{t}{R}+2-2 \cos \theta\right)^{3}} d \theta \\
f_{1}=\int_{-\theta_{m}}^{\theta_{m}} \frac{\cos \theta}{\left(\frac{t}{R}+2-2 \cos \theta\right)^{3}} d \theta=\frac{8 \gamma^{4}(2 \gamma+1) \tan \frac{\theta_{m}}{2}}{(4 \gamma+1)^{2}\left[1+(4 \gamma+1) \tan ^{2} \frac{\theta_{m}}{2}\right]^{2}}+ \\
\frac{4 \gamma^{3}\left(6 \gamma^{3}+3 \gamma+1\right) \tan \frac{\theta_{m}}{2}}{(4 \gamma+1)^{2}\left[1+(4 \gamma+1) \tan ^{2} \frac{\theta_{m}}{2}\right]}+\frac{12 \gamma^{4}(2 \gamma+1)}{(4 \gamma+1)^{5 / 2}} \arctan \left(\sqrt{4 \gamma+1} \tan \frac{\theta_{m}}{2}\right)
\end{gathered}
$$


Where the parameters $r$ can be expressed by Eq.3.

$$
\gamma=\frac{R}{t}
$$

The rotation rigidity formula of arc flexible hinge is given by Eq.4.

$$
K_{1}=\frac{M_{Z}}{\alpha_{Z}}=\frac{E b R^{2}}{12 f_{1}}
$$

\section{FEA Model of Arc Flexible Hinge}

ANSYS has some characteristics that the general finite element analysis technology, powerful computing, and reliable result. The arc flexible hinge's basic structure size is $\mathrm{b}=10 \mathrm{~mm}, \mathrm{t}=1 \mathrm{~mm}, \mathrm{R}=2.5 \mathrm{~mm}, \theta_{\mathrm{m} .}=90^{\circ}$ The material is Spring steel, $\mathrm{E}=200 \mathrm{GPa}$, $v=0.3$.

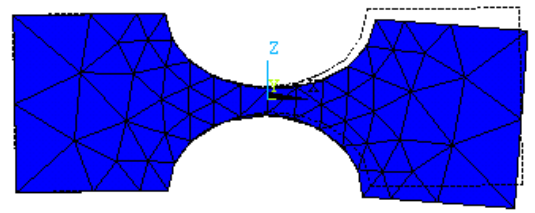

Fig. 2. FEA model of arc flexure hinge

The arc flexible hinge FEA model is shown in Figure 2.The model left end surface is restrained completely; the right end surface exerts bending moment $M=0.1 \mathrm{~N} . \mathrm{m}$. The special node 1 of the grid model right end surface represents the output displacement. The unit type chooses 3-D the entity SOLID92 unit model, the entire model uses smart size to free mesh. The FEA model and the output displacement have been obtained with changing arc flexible hinge's parameters $\mathrm{E}, \mathrm{b}, \mathrm{t}$ and $\mathrm{R}$ as well as $\theta_{m}$.

\section{Performance Analysis of Stiffness}

The theoretical calculation and FEA rotational stiffness are obtained through changing arc flexure hinge parameters $E 、 b 、 t$ and $R$ as well as $\theta_{m}$, as shown in Figure 3 to7. The theoretical value of arc flexible hinge stiffness is simplified theory. FEA is FEA value of arc flexible hinge stiffness. 


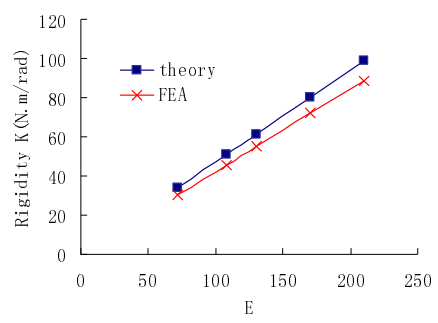

Fig. 3. Comparison of FEA value and the theoretical value of flexure hinge with changing $\mathrm{E}$

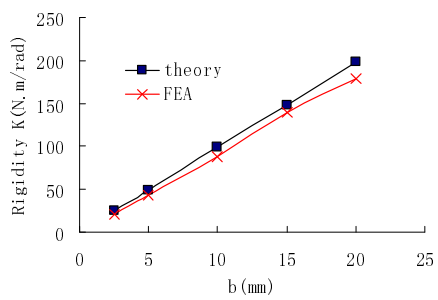

Fig. 4. Comparison of FEA value and the theoretical value of flexure hinge with changing width $b$

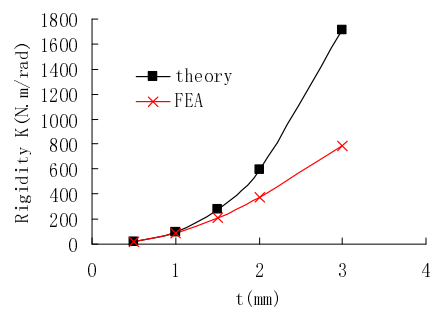

Fig. 5. Comparison of FEA value and the theoretical value of flexure hinge with changing thickness $\mathrm{t}$

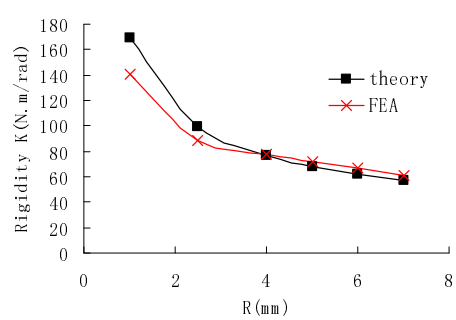

Fig. 6. Comparison of FEA value and the theoretical value of flexure hinge with changing radius $R$ 


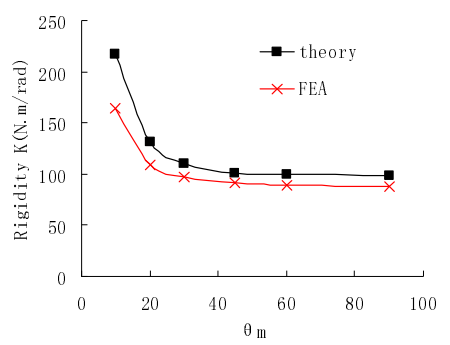

Fig. 7. Comparison of FEA value and the theoretical value of flexure hinge with changing centre angle $\theta_{m}$

From Eq.1, Eq.2and Figures 3 to 7 the following conclusions can be observed.

(1)From Figures 3 and 4 it can be observed that the rotation stiffness is a linearly increasing relation with material young's modulus $\mathrm{E}$ and width $\mathrm{b}$. The theoretical value is bigger than the FEA value. When $\mathrm{E}$ and $\mathrm{b}$ are smaller, the FEA value and the theoretical value are closer.

(2) From Figure 5 it can observed that the rotation of the stiffness FEA value and the theoretical value is a curve increasing with thickness $t$, end the speed-up is getting quicker and quicker. When $t$ is bigger, the difference of the theoretical value and the FEA value is bigger. When $\mathrm{t}<2 \mathrm{~mm}$, the FEA value and the theoretical value are close.

(3) From Figure 6 it can be observed that the relation of rotation rigidity and radius $\mathrm{R}$ is a decreasing curve, and the rate of reduced scope is gradually decreased. When $\mathrm{R}>2 \mathrm{~mm}$, the FEA value and the theoretical value are closer.

(4) From Figure7 it can be observed that the relation of rotation rigidity and centre angle $\theta_{\mathrm{m}}$ is a decreasing curve, but the decreasing scope is small. The theoretical value is bigger than the FEA value. When $\theta_{\mathrm{m}}>20^{\circ}$ the FEA value and the theoretical value are closer.

From Figures 3 to 7 it can be observed that the influence of flexure hinge's parameter to its rotation stiffness is: the influence of thickness $t$ is biggest, followed by radius $\mathrm{R}$, centre angle $\theta_{\mathrm{m}}$, width $\mathrm{b}$ and $\mathrm{E}$.

The theoretical value and the FEA value of an arc flexible hinge rotation rigidity is not equal, even if has a big differential value. The reasons are:

(1) The flexible hinge theoretical model that is established using materials mechanics' bending strain theory is built on the basis of certain assumptions.

(2) From Figure 2 it can be observed that the FEA model not only has the displacement in the $\mathrm{y}$ axis direction, but also has the displacement change on the $\mathrm{z}$ axis direction, when the torque $M_{z}$ exerted on the $\mathrm{z}$ axis for the model. In other words, the flexible hinge not only has the bending strain, but also will have the shearing force to cause upward deformation. Under certain design parameters, the flexible hinge's theory and FEA solution achieve a good match. 


\section{Conclusions}

The different design parameters to the flexible hinge rotation rigidity influence and the linear relationship are obtained by comparing the rotation rigidity theory solution and the finite element analysis. It can be observed that the influence of flexible hinge's parameter to its rotation stiffness is: the influence of thickness $t$ is biggest, followed by radius $\mathrm{R}$, centre angle $\theta_{\mathrm{m}}$, width $\mathrm{b}$ and $\mathrm{E}$. The reasons that the theoretical value and the FEA value of an arc flexible hinge's rotation rigidity is not equal is that the flexible hinge not only has the bending strain, but also will have the shearing force to cause upward deformation. It is helpful to further analyze the movement of the mechanical deformation mechanism, using the finite element technology to simulate the flexible hinge performance.

Acknowledgments. This research is financially supported by the Hangzhou Binteng Technology Co., LTD, Hangzhou Province, China.

\section{References}

1. Zuo, X.Y., Liu, X.M.: Calculation and analysis of rotational stiffness for three types of flexure hinges. Chinese Journal of Scientific Instrument 27, 1725-1728 (2006)

2. Lobontiu, N., Garcia, E.: Analytical model of displacement amplification and stiffness optimization for a class of flexure-based compliant mechanisms. Computer \& Stuctures 81 , 2797-2801 (2003)

3. Paros, J.M., Weisibord, L.: How to design flexure hinge. Machine Design 37, 151-157 (1965)

4. Smith, T.S., Badami, V.G.: Arc flexure hinges. Review Scientific Instruments 68, 1474-1483 (1997)

5. Lobontiu, N., Paine, J.S.N.: Corner-filleted flexure hinges. Journal of Mechanical Design 123, 346-352 (2001)

6. Xu, W., King, T.G.: Flexure hinges for piezo-actuator displacement amplifiers: flexibility, accuracy and stress considerations. Precision Engineering 19, 4-10 (2002)

7. Abdellatif, H., Benimeli, F., Heimann, B., Grotjahn, M.: Direct identification of dynamic parameters for parallel manipulators. Presented at International Conference on Mechatronics and Robotics, MechRob 2004, Aachen, Germany (2004) 